\title{
Manuels de Pierre Schulz
}

\section{Pierre Sindelar}

Dr méd., spécialiste en psychiatrie, membre de la FMH

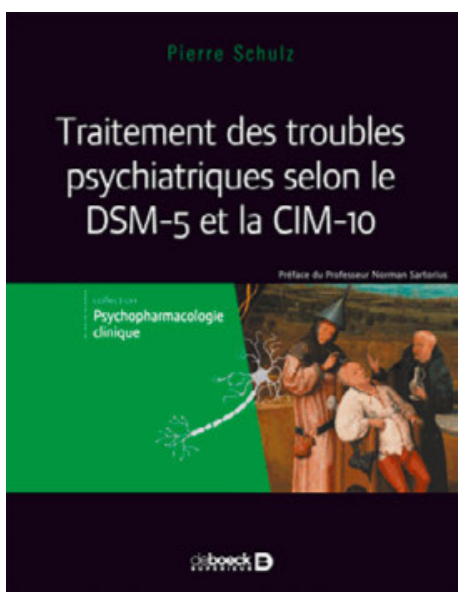

Pierre Schulz et Norman Sartorius (Prefacier) Traitement des troubles psychiatriques selon le DSM-5 et la CIM-10 (Volume 3)

De Boeck supérieur, 2016

1394 pages. $124.60 \mathrm{CHF}$.

ISBN 978-2-80730-306-5

J'aimerais signaler trois publications aux nombreux lecteurs qui s'intéressent aux neurosciences cliniques, à la psychopharmacologie et aux traitements en psychiatrie.

Le docteur Pierre Schulz, qui a travaillé en psychiatrie et en psychopharmacologie dans les Hôpitaux Universitaires de Genève (HUG), a publié récemment le troisième volume d'une collection nommée Psychopharmacologie clinique. Ce volume est intitulé Traitement des troubles psychiatriques selon le DSM-5 et la CIM-10, un texte de 1400 pages (donc au-delà des 1100 pages de la version française du DSM-5) qui reprend les entités cliniques du DSM- 5 sans toutefois citer le texte du DSM-5. Il ajoute à cette bible de la psychiatrie, comme certains ont nommé le DSM-5, les données suivantes: comparaison des critères de classification CIM-10 avec ceux du DSM-5, mention des projets pour la CIM-11, épidémiologie de la comorbidité de chaque trouble, troubles non inclus dans le DSM-5, psychopathologie et physiopathologie de chaque trouble, données quantifiées quant aux résultats thérapeutiques avec les traitements non médicamenteux et médicamenteux, questions et controverses et pro- positions résumées de diagnostic, information au patient et prise en soins. Ce manuel ne fait donc pas double emploi avec le DSM-5.

\section{Un confort de lecture}

Lors de son activité aux Hôpitaux universitaires de Genève (HUG), avant sa retraite, cet auteur avait déjà transmis avec succès dans son enseignement (notamment au travers de consultations données par l'Unité de psychopharmacologie dont il était responsable) la capacité de critiquer les publications de recherche clinique en psychiatrie. Il se montrait un conseiller utile et avisé pour les cliniciens dans le choix des traitements et la compréhension des effets indésirables ou des interactions.

Le fait qu'il s'agit d'un livre rédigé par un seul auteur fait que l'on retrouve les mêmes thèmes traités dans le même ordre dans chaque chapitre, ce qui apporte un confort de lecture par rapport à des séries de chapitres spécialisés reflétant l'intérêt et les travaux d'une multitude d'auteurs. La rédaction de ce manuel a toutefois bénéficié des critiques et commentaires de collègues cliniciens et chercheurs, ce qui apporte une forme de validation de cette somme assez conséquente d'informations.

Ce dernier opus se présente donc comme une approche généraliste de la psychiatrie, avec des notions spécialisées comme la neuropsychologie, la physiopathologie, la neuroimagerie et les résultats d'essais cliniques de traitements pas les psychotropes ou d'autres méthodes.

\section{Il ajoute des données supplémentaires} à cette bible de la psychiatrie, comme certains ont nommé le DSM-5.

\section{Des questions et des controverses}

Le diagnostic et le traitement des troubles mentaux furent approchés différemment depuis les années 1990 avec le début de la médecine fondée sur des preuves, à savoir que les types de preuves ont été passés au crible de l'expertise scientifique et statistique et les conclusions diffusées en espérant par là diminuer le nombre de décisions prises sans respect de ces faits. Cela étant, 
le psychiatre, le psychologue clinicien et plus largement tout médecin est amené à répondre à une demande de soins qui peut souvent, contrairement à la médecine somatique, ne pas référer à des symptômes évoquant un trouble ou une maladie précise: ces personnes reçoivent des soins en absence d'un diagnostic précis, ce qui soulève, outre des problèmes méthodo-

\section{La rédaction de ce manuel a toutefois bénéficié des critiques et commentaires de collègues cliniciens et chercheurs.}

logiques, la question d'une médicalisation de la société. Ces questions et d'autres sont abordées en fin de chaque chapitre dans des paragraphes décrivant des questions et des controverses, donc soulignant que les connaissances acquises ne sont ni complètes ni définitives!

Le lecteur lira aussi avec intérêt les deux volumes antérieurs de la collection Psychopharmacologie clinique.
Le premier, Psychiatrie et neuroscience (550 pages, publié en 2012), offre une synthèse des données acquises récemment dans les sciences fondamentales et cliniques en regard de la psychiatrie. Il contient des chapitres de synthèse sur les fonctions supérieures (émotions, mémoire, etc.), sur la neuroimagerie, la génétique, l'épigénétique, la psychologie de l'évolution et la méthodologie de la recherche, dont la statistique et les essais cliniques. Le deuxième volume Traitements biologiques en psychiatrie (790 pages, publié en 2013), décrit quant à lui en détails les traitements biologiques et non biologiques.

Je reconnais à ces trois volumes une grande pertinence pour la pratique clinique, ce dont le lecteur peut se faire sa propre idée en allant consulter le portail Internet de vente de livres Decitre où l'on trouve en accès libre une cinquantaine des premières pages de ces trois volumes, qui ont je pense amplement leur place dans la bibliothèque des psychiatres et des psychologues cliniciens. 